\title{
EXCITATION OF MANTLE RAYLEIGH WAVES OF PERIOD 100 SECONDS AS A FUNCTION OF MAGNITUDE
}

\author{
By James N. Brune and ChI-Yu Krng*
}

\begin{abstract}
The excitation of mantle Rayleigh waves of 100 seconds period as a function of magnitude is studied using data from 91 earthquakes in the magnitude range 5.0 to 8.9. The data were recorded on a wide variety of instruments including Milne-Shaw horizontal pendulums and modern long-period high-gain inertial seismographs. The larger earthquakes studied range in time from 1923 to 1964. Mantle Rayleigh wave amplitudes are corrected to a distance of $90^{\circ}$ and plotted as a function of surface wave magnitude. The data are compared with theoretical curves based on a moving source model and two statistical models discussed by Aki. It is concluded that for large earthquakes the source may be approxımated by a point couple which propagates a distance given approximately by the length of the aftershock zone.
\end{abstract}

\section{INTRODUCTION}

Large shallow earthquakes generate fundamental mode Rayleigh waves of periods greater than 90 seconds-herein called mantle Rayleigh waves-with sufficient amplitude to be recorded on modern long-period inertial seismographs and strain meters. It is less well-known that the largest shallow earthquakes generate mantle waves large enough to be clearly recorded on Milne-Shaw and Wiechert horizontal component mechanical pendulums which have been in operation since the early 1900's. Recently developed long-period seismographs make recording of mantle waves possible for earthquakes of magnitudes as low as 5. Unfortunately, with many older instruments being replaced by new instruments with high magnification it is now difficult to find instruments which stay on scale during very large earthquakes.

In this paper we report on a study of the excitation of mantle waves as a function of magnitude. Measurements are made on several different types of seismographs capable of recording mantle waves. The results are interpreted in terms of the expected excitation function based on Tocher's and Iida's curves for fault length versus magnitude.

\section{INSTRUMENTS}

Instruments of several types were used to record the mantle waves studied in this paper. These are summarized in Table 1 which gives instrument designation. and gain at a period of 100 seconds.

The Milne-Shaw horizontal mechanical pendulums have a free period of 12 seconds. The data recorded on these instruments were from Canadian stations operat-

* Now at the Institute of Geophysics and Planetary Physics, University of California, Los Angeles. 
ing since 1923. The Wiechert horizontal pendulums are similar but with a free period of 8-9 seconds. Strain seismographs are those developed by Benioff (1959).

Columbia-IGY instruments are electromagnetic seismographs with pendulum period 15 seconds and galvanometer period 80 seconds. These instruments are described by Sutton and Oliver (1959). WWSSN instruments are the long-period electromagnetic seismograph of the World-Wide Standardized Seismograph Network. These instruments are described in publications of the U. S. Coast and Geodetic Survey.

The CIT ULP instruments are ultra-long period seismographs (peak response at a

TABLE 1

INSTRUMENTS

\begin{tabular}{cllr}
\hline Earthquake No. & \multicolumn{1}{c}{ Stations } & \multicolumn{1}{c}{ Instrument } & $\begin{array}{c}\text { Amplification } \\
\text { at } 100 \mathrm{sec}\end{array}$ \\
\hline $1-16$ & VIC, TNT, OTT & Milne-Shaw & 4 \\
9 & PAS & Strain & 30 \\
$15 \mathrm{a}$ & PAS & Long Period Benioff & 25 \\
16 & PAS & Strain & 4 \\
$17,18,19$ & RDJ, SUV & Columbia-IGY & 150 \\
& MTJ, PER, OTT, UPP & Columbia-IGY & 70 \\
$18 \mathrm{a}$ & PAS & Strain & 4.4 \\
$19 \mathrm{a}$ & BER, JEN & Wiechert & 1.5 \\
& KIP & Strain & 28 \\
$20-25$ & PAL, HON & Columbia- & 60 \\
& & ULP filtered & \\
25 & JER & WWSSN & 416 \\
& ESK & WWSSN & 208 \\
26 & OGD & WWSSN & 833 \\
27 & KTG, HKC & WWSSN & 208 \\
& GOL & WWSSN & 416 \\
$28-33$ & ISA & CIT-ULP & 500 \\
$34-44$ & PAS & CIT-ULP & 500 \\
$45-88$ & PAS & CIT-ULP & 1600 \\
\hline
\end{tabular}

period of 80 seconds) with capacitor transducers and are described by Gilman (1960). The Columbia ULP filtered instruments are long-period electromagnetic seismographs using filter galvanometers to reject surface waves of periods less than 70 sec. These are described by Brune (1963).

\section{Data Analusis}

A total of 91 earthquakes with magnitudes ranging from õ to 8.9 were investigated in this study. Origin times, epicenters, depths, magnitudes, recording stations and approximate epicentral distances are listed in Table 2. The magnitude used in this study is $M_{s}$ as defined by Gutenberg (1945) and is primarily based on the amplitudes of 20 second surface waves; however, it may be determined from body waves also (Gutenberg and Richter, 1956; Richter, 1958). Most magnitudes less than 7.5 were determined at Pasadena. Magnitudes greater than 7.5 were deter- 
TABLE 2

\begin{tabular}{|c|c|c|c|c|c|c|c|c|c|c|c|c|}
\hline \multirow{2}{*}{ 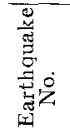 } & \multirow{2}{*}{ Date } & \multicolumn{3}{|c|}{ Origin Time } & \multirow{2}{*}{ Lat. } & \multirow{2}{*}{ Long. } & \multirow{2}{*}{$\begin{array}{l}\text { Depth } \\
(\mathrm{km})\end{array}$} & \multirow{2}{*}{$\begin{array}{l}\text { Magni- } \\
\text { tude }\end{array}$} & \multirow{2}{*}{ Station } & \multirow{2}{*}{$\begin{array}{c}\text { Epi- } \\
\text { central } \\
\text { Distance } \\
\text { (degrees) }\end{array}$} & \multirow{2}{*}{$\begin{array}{c}\text { Signal } \\
\text { Used }\end{array}$} & \multirow{2}{*}{$\begin{array}{l}\text { Log } \\
A_{90}{ }^{*}\end{array}$} \\
\hline & & h & $\mathrm{m}$ & $\mathbf{s}$ & & & & & & & & \\
\hline 1 & Feb. 3, 1923 & 16 & 01 & 41 & $54 \mathrm{~N}$ & $161 \mathrm{E}$ & & 8.4 & $\mathrm{VIC}$ & 46 & $\mathrm{R}_{2}$ & $3.37+$ \\
\hline \multirow[t]{2}{*}{2} & June 26,1924 & 01 & 37 & 34 & $56 \mathrm{~S}$ & $157 \frac{\mathrm{t}}{2} \mathrm{E}$ & & 8.3 & VIC & 120 & & \\
\hline & & & & & & & & & & & $\begin{array}{l}\mathrm{R}_{2} \\
\mathrm{R}_{3}\end{array}$ & $\begin{array}{l}3.01 \dagger \\
3.50 \dagger\end{array}$ \\
\hline 3 & June 17,1928 & 08 & 19 & 27 & $16 \frac{1}{2} \mathrm{~N}$ & $98 \mathrm{~W}$ & & 7.9 & $\mathrm{VIC}$ & 38 & $R_{2}$ & $3.62 \dagger$ \\
\hline 4 & June 27, 1929 & 12 & 47 & 05 & $54 \mathrm{~S}$ & $29 \frac{1}{2} W$ & & 8.3 & VIC & 132 & $\mathrm{R}_{2}$ & $2.79 \dagger$ \\
\hline 5 & Jan. 15, 1931 & 01 & 50 & 41 & $39 \frac{\mathrm{I}}{2} \mathrm{~S}$ & $177 \mathrm{E}$ & & 7.9 & VIC & 104 & $\mathrm{R}_{2}$ & $2.43 \dagger$ \\
\hline 6 & May 14,1932 & 13 & 11 & 00 & $\frac{1}{2} \mathrm{~N}$ & $126 \mathrm{E}$ & & 8.3 & VIC & 102 & $\mathrm{R}_{2}$ & $3.01 \uparrow$ \\
\hline \multirow[t]{2}{*}{7} & June 3, 1932 & 10 & 36 & 50 & $19 \frac{1}{2} \mathrm{~N}$ & $104 \frac{1}{2} W$ & & 8.1 & TNT & 32 & $\mathrm{R}_{2}$ & $2.66 \dagger$ \\
\hline & & & & & & & & & VIC & 32 & $\mathrm{R}_{2}$ & $3.27 \dagger$ \\
\hline \multirow[t]{2}{*}{8} & June 18, 1932 & 10 & 12 & 10 & $19 \frac{1}{2} \mathrm{~N}$ & $103 \frac{1}{2} W$ & & 7.9 & TNT & 32 & $\mathrm{R}_{2}$ & $2.36 \dagger$ \\
\hline & & & & & & & & & VIC & 32 & $\mathrm{R}_{2}$ & $2.23 \dagger$ \\
\hline 9 & March 2, 1933 & 17 & 30 & 54 & $39 \frac{1}{4} \mathrm{~N}$ & $144 \frac{1}{2} \mathrm{E}$ & & 8.9 & VIC & 61 & $\mathrm{R}_{2}$ & $3.10 \dagger$ \\
\hline & & & & & & & & & PAS & 75 & $R_{2}$ & $3.53 \hbar$ \\
\hline 10 & Jan. 15, 1934 & 08 & 43 & 18 & $26 \frac{1}{2} \mathrm{~N}$ & $86 \frac{1}{3} \mathrm{E}$ & & 8.4 & VIC & 100 & $\mathrm{R}_{2}$ & $2.77 \dagger$ \\
\hline 11 & July 18,1934 & 19 & 40 & 15 & $11^{\frac{3}{2}} \mathrm{~S}$ & $166 \frac{1}{2} \mathrm{E}$ & & 8.1 & TNT & 118 & $\mathrm{R}_{2}$ & $2.60 \dagger$ \\
\hline 12 & Sept. 20,1935 & 01 & 46 & 33 & $11^{\frac{3}{4}} \mathrm{~S}$ & $166 \frac{\mathrm{I}}{2} \mathrm{E}$ & & 7.9 & TNT & 118 & $R_{1}$ & $3.05 \dagger$ \\
\hline & & & & & & & & & $\mathrm{VIC}$ & 86 & $R_{l}$ & $2.73 \dagger$ \\
\hline 13 & Dec. 28,1935 & 02 & 35 & 22 & 0 & $98 \frac{1}{4} \mathrm{E}$ & & 8.1 & TNT & 137 & $R_{2}$ & $2.45 \dagger$ \\
\hline 14 & Nov. 25, 1941 & 18 & 03 & 55 & $37 \frac{1}{2} \mathrm{~N}$ & $18 \frac{1}{2} W$ & & 8.4 & TNT & 44 & $R_{2}$ & $2.93 \dagger$ \\
\hline 15 & Nov. 10,1942 & 11 & 41 & 27 & $49 \frac{1}{3} \mathrm{~S}$ & $32 \mathrm{E}$ & & 8.3 & VIC & 160 & $\mathrm{R}_{3}$ & $3.44 \dagger$ \\
\hline $15 a$ & Aug. 15,1950 & 14 & 09 & 30 & $28.6 \mathrm{~N}$ & $96.5 \mathrm{E}$ & & 8.6 & PAS & 110 & $\mathrm{R}_{2}$ & $3.40 \ddagger$ \\
\hline 16 & Nov. 4,1952 & 16 & 58 & 26 & $523 \mathrm{~N}$ & $159 \frac{1}{2} \mathrm{E}$ & & 8.4 & OTT & 73 & $\mathrm{R}_{3}$ & $3.55 \dagger$ \\
\hline & & & & & & & & & PAS & 59 & $\mathrm{R}_{2}$ & $3.74 \ddagger$ \\
\hline 17 & July 10,1958 & 06 & 15 & 54 & $58 \frac{1}{2} \mathrm{~N}$ & $136 \mathrm{~W}$ & & $7 \frac{9}{4}-8$ & RDJ & 110 & $\mathrm{R}_{2}$ & $2.24 \div$ \\
\hline & & & & & & & & & PER & 125 & $\mathbf{R}_{1}$ & 2.32 \\
\hline & & & & & & & & & OTT & 37.8 & $\mathrm{R}_{2}$ & 2.64 \\
\hline & & & & & & & & & UPP & 59.7 & $\mathrm{R}_{2}$ & 2.55 \\
\hline 18 & Sept. 4, 1958 & 21 & 51 & 08 & $33 \frac{1}{2} \mathrm{~S}$ & $69 \frac{1}{2} W$ & & $6 \frac{3}{4}-7$ & MTJ & 150 & $\mathrm{R}_{1}$ & 1.41 \\
\hline $18 a$ & May 22,1960 & 19 & 11 & 20 & $38 \mathrm{~S}$ & $73 \frac{1}{2} W$ & & 8.4 & PAS & 82 & $\mathrm{R}_{2}$ & $3.42 \dagger$ \\
\hline 19 & Aug. 9, 1960 & 07 & 39 & 23 & $40 \mathrm{~N}$ & $126.6 \mathrm{~W}$ & 25 & 6 & SUV & 76 & $\mathrm{R}_{\mathrm{i}}$ & 1.06 \\
\hline $19 \mathrm{a}$ & March 28, 1964 & 08 & 37 & 14.2 & $61.1 \mathrm{~N}$ & $147.7 \mathrm{~W}$ & 33 & 8.4 & $\begin{array}{l}\text { JEN } \\
\text { BER }\end{array}$ & & $\begin{array}{l}\mathrm{R}_{2} \\
\mathrm{R}_{2}\end{array}$ & 4.00 \\
\hline & & & & & & & & & KIP & & $\mathrm{R}_{i}$ & $2.96 t$ \\
\hline & & & & & & & & & & & $\mathrm{R}_{3}$ & $3.42 \ddagger$ \\
\hline 20 & Nov. 19,1904 & 23 & 35 & 06 & $6.0 \mathrm{~S}$ & $150.8 \mathrm{E}$ & 3 & $6 \frac{\hat{3}}{4}$ & PAI & 127.1 & $\mathrm{R}_{1}$ & 1.50 \\
\hline & & & & & & & & & & & $\mathrm{R}_{2}$ & 1.44 \\
\hline 21 & Jan. 24, 1965 & 00 & 11 & 12 & $2.4 \mathrm{~S}$ & $126.0 \mathrm{E}$ & 6 & $7 \frac{1}{2}-7 \frac{3}{1}$ & PAL & 137.6 & $\mathrm{R}_{1}$ & 2.66 \\
\hline & & & & & & & & & & & $\mathrm{R}_{2}$ & 2.81 \\
\hline & & & & & & & & & & & $\mathrm{R}_{3}$ & 2.93 \\
\hline & & & & & & & & & $\mathrm{HON}$ & 77.8 & $\mathrm{R}_{2}$ & 3.10 \\
\hline 22 & Feb. 4, 1965 & 05 & 01 & 22 & $51.3 \mathrm{~N}$ & $178.6 \mathrm{E}$ & 40 & $7 \frac{3}{4}$ & HON & 35.0 & $\mathrm{R}_{3}$ & 2.76 \\
\hline 23 & March 21, 1965 & 11 & 08 & 16 & $1.5 \mathrm{~S}$ & $126.5 \mathrm{E}$ & 33 & $6 \frac{3}{4}$ & PAL & 136.6 & $\mathrm{R}_{\mathrm{I}}$ & 1.35 \\
\hline 24 & March 28, 1965 & 16 & 33 & 15 & $32.4 \mathrm{~S}$ & $71.2 \mathrm{~W}$ & 72 & $7-7 \frac{1}{4}$ & $\mathrm{PAL}_{2}$ & 73.1 & $R_{1}$ & 1.67 \\
\hline & & & & & & & & & $\mathrm{HON}$ & 98.6 & $\mathrm{R}:$ & 2.15 \\
\hline 25 & March 30,1965 & 02 & 27 & 07 & $50.6 \mathrm{~N}$ & $177.9 \mathrm{E}$ & 51 & $7-7 \frac{1}{4}$ & PAL & 69.3 & $\mathrm{R}_{2}$ & 2.41 \\
\hline & & & & & & & & & HON & 34.8 & $\mathbf{R}_{1}$ & 2.01 \\
\hline & & & & & & & & & JER & 90 & $\mathrm{R}_{\tilde{s}}$ & 2.43 \\
\hline & & & & & & & & & ESK & 72 & $\mathrm{R}_{3}$ & 2.65 \\
\hline 26 & May 20, 1965 & 00 & 40 & 11 & $14.7 \mathrm{~S}$ & $167.4 \mathrm{E}$ & 16 & $6 \frac{3}{4}-7$ & OGD & 120 & $\mathrm{R}_{3}$ & 2.03 \\
\hline 27 & Aug. 2, 1965 & 13 & 19 & 55 & $56.2 \mathrm{~S}$ & $158.2 \mathrm{E}$ & 33 & $7-7 \frac{1}{4}$ & $\mathrm{KTG}$ & 158 & $\mathrm{R}_{3}$ & 1.89 \\
\hline & & & & & & & & & $\mathrm{HKC}$ & 81 & $\mathrm{R}_{2}$ & 2.14 \\
\hline & & & & & & & & & & & $\mathrm{R}_{3}$ & 2.31 \\
\hline & & & & & & & & & GOL & 98 & $\mathbf{R}_{3}$ & 1.94 \\
\hline 28 & Aug. 23, 1965 & 18 & 46 & 02 & $16.3 \mathrm{~N}$ & $95.8 \mathrm{~W}$ & 20 & $7 \frac{1}{4}$ & ISA & 27.9 & $\mathbf{R}_{2}$ & 1.63 \\
\hline & & & & & & & & & & & $\mathrm{R}_{3}$ & 1.87 \\
\hline 29 & Sept. 4,1965 & 14 & 32 & 47 & $58.2 \mathrm{~N}$ & $152.7 \mathrm{~W}$ & 10 & $6 \frac{3}{4}-7$ & ISA & 31.9 & $\mathbf{R}_{1}$ & 1.37 \\
\hline
\end{tabular}

* A = Vertical ground amplitude in microns for Rayleigh waves with periods of approximately 100 seconds.

$\uparrow$ Value of A computed from horizontal trace amplitudes assuming $A_{Z} / A_{H}=1.3$.

+ Value of A computed from strain seismograph. 
TABLE 2-Continued

\begin{tabular}{|c|c|c|c|c|c|c|c|c|c|c|c|c|}
\hline \multirow{2}{*}{ 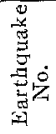 } & \multirow{2}{*}{ Date } & \multicolumn{3}{|c|}{ Origin Time } & \multirow{2}{*}{ Lat. } & \multirow{2}{*}{ Long. } & \multirow{2}{*}{$\underset{(\mathrm{km})}{\text { Depth }}$} & \multirow{2}{*}{$\begin{array}{c}\text { Magni- } \\
\text { tude }\end{array}$} & \multirow{2}{*}{ Station } & \multirow{2}{*}{$\begin{array}{c}\text { Epi- } \\
\text { central } \\
\text { Distance } \\
\text { (degrees) }\end{array}$} & \multirow{2}{*}{$\mid \begin{array}{c}\text { SignaI } \\
\text { Used }\end{array}$} & \multirow{2}{*}{$\begin{array}{l}\log _{90} \\
A_{90}\end{array}$} \\
\hline & & $\mathrm{h}$ & $\mathrm{m}$ & s & & & & & & & & \\
\hline 30 & Sept. 12, 1965 & 08 & 40 & 11 & $6.4 \mathrm{~S}$ & $151.6 \mathrm{E}$ & 29 & $8 \frac{1}{2}-6 \frac{3}{x}$ & ISA & 93.6 & $\mathrm{R}_{1}$ & 0.13 \\
\hline 31 & Sept. 17,1965 & 11 & 13 & 56 & $1.5 \mathrm{~S}$ & $77.7 \mathrm{~W}$ & 191 & $6 \frac{1}{2}$ & ISA & 53.0 & $\mathbf{R}_{1}$ & 0.59 \\
\hline 32 & Sept. 17,1965 & 16 & 21 & 19 & $36.3 \mathrm{~N}$ & $141.3 \mathrm{E}$ & 40 & $6 \frac{3}{4}$ & ISA & 77.0 & $\mathrm{R}_{3}$ & 1.02 \\
\hline 33 & Sept. 21, 1965 & 01 & 38 & 31 & $29.0 \mathrm{~N}$ & $128.1 \mathrm{E}$ & 199 & $6 \frac{3}{4}$ & ISA & 90.2 & $R_{1}$ & 1.19 \\
\hline 34 & Sept. 30,1965 & 23 & 47 & 40 & $59.7 \mathrm{~N}$ & $143.4 \mathrm{~W}$ & 12 & 5.3 & PAS & 30.5 & $\mathbf{R}_{1}$ & 0.46 \\
\hline 35 & Oct. 1,1965 & 08 & 52 & 06 & $50.1 \mathrm{~N}$ & $178.3 \mathrm{E}$ & 32 & $6 \frac{1}{2}$ & PAS & 48.3 & $\mathrm{R}_{1}$ & 0.55 \\
\hline 36 & Oct. 3, 1965 & 14 & 45 & 27 & $49.5 \mathrm{~N}$ & $156.5 \mathrm{E}$ & $33 \mathrm{R}$ & 6 & PAS & 62.1 & $\mathrm{R}_{1}$ & 0.01 \\
\hline 37 & Oct. 3,1965 & 16 & 14 & 55 & $42.9 \mathrm{~S}$ & $75.4 W$ & 28 & $6 \frac{1}{2}$ & PAS & 86.1 & $\mathbf{R}_{\mathbf{i}}$ & $0.7 i$ \\
\hline 38 & Oct. 12,1965 & 13 & 40 & 56 & $56.3 \mathrm{~N}$ & $153.7 \mathrm{~W}$ & 11 & 5.2 & PAS & 32.8 & $\mathrm{R}_{1}$ & -0.17 \\
\hline \multirow[t]{2}{*}{39} & Oct. 18, 1965 & 21 & 60 & 05 & $1.1 \mathrm{~S}$ & $127.9 \mathrm{E}$ & $33 \mathrm{R}$ & $6 \frac{3}{4}$ & PAS & 110.3 & $\mathrm{R}_{\mathrm{t}}$ & 0.95 \\
\hline & & & & & & & & & & & $\mathrm{R}_{9}$ & 1.12 \\
\hline 40 & Oct. $19, \mathbf{1 9 6 5}$ & 20 & 48 & 47 & $52.3 \mathrm{~N}$ & $174.3 \mathrm{E}$ & $48 \mathrm{R}$ & 5.5 & PAS & 50.5 & $\mathbf{R}_{\mathrm{t}}$ & -0.05 \\
\hline 41 & Nov. 6,1965 & 09 & 21 & 49 & 22.I S & $113.8 \mathrm{~W}$ & $33 \mathrm{R}$ & 6 & PAS & 56.1 & $\mathrm{R}_{1}$ & 0.39 \\
\hline 42 & Nov. 12,1965 & 17 & 52 & 24 & $30.5 \mathrm{~N}$ & $140.2 \mathrm{E}$ & 40 & $6 \frac{3}{4}$ & PAS & 82.1 & $\mathrm{R}_{1}$ & 0.64 \\
\hline & & & & & & & & & & & $\mathrm{R}_{2}$ & 0.75 \\
\hline 43 & Nov. 13,1965 & 04 & 33 & 53 & $43.8 \mathrm{~N}$ & $87.8 \mathrm{E}$ & $59 \mathrm{R}$ & $6 \frac{3}{4}$ & PAS & 98.9 & $R_{1}$ & 0.76 \\
\hline 44 & Nov. 15,1865 & 11 & 18 & 50 & $0.3 \mathrm{~S}$ & $18.7 \mathrm{~W}$ & $24 \mathrm{R}$ & $6 \frac{1}{2}$ & PAS & 97.7 & $\mathbf{R}_{1}$ & 1.41 \\
\hline & & & & & & & & & & & $\mathrm{R}_{2}$ & 1.18 \\
\hline 45 & Nov. 16,1965 & 15 & 24 & 43 & $31.0 \mathrm{~N}$ & $41.5 \mathrm{~W}$ & $17 \mathrm{R}$ & $6 \frac{1}{2}$ & PAS & 63.2 & $\mathrm{R}_{1}$ & 0.67 \\
\hline & & & & & & & & & & & $\mathrm{R}_{2}$ & 0.96 \\
\hline 46 & Nov, 19,1965 & 07 & 14 & 13 & $45.3 \mathrm{~N}$ & $150.9 \mathrm{E}$ & 13 & 5.5 & PAS & 67.3 & $\mathbf{R}_{\mathrm{I}}$ & 0.05 \\
\hline 47 & Nov, 21,1965 & 10 & 31 & 50 & $6.1 \mathrm{~S}$ & $130.4 \mathrm{E}$ & 93 & 6 & PAS & 111.1 & $\mathbf{R}_{I}$ & 0.94 \\
\hline & & & & & & & & & & & $\mathrm{R}_{2}$ & 0.87 \\
\hline 48 & Dec. 6,1965 & 11 & 34 & 54 & $18.9 \mathrm{~N}$ & $107.1 \mathrm{~W}$ & 37 & $6 \frac{3}{4}$ & PAS & 18.1 & $\mathbf{R}_{2}$ & 1.26 \\
\hline & & & & & & & & & & & $\mathbf{R}_{3}$ & 1.07 \\
\hline 49 & Dec. 6,1965 & 18 & 42 & 33 & $18.8 \mathrm{~N}$ & $107.0 \mathrm{~W}$ & 40 & $5 \frac{3}{4}$ & PAS & 18.2 & $\mathbf{R}_{1}$ & -0.37 \\
\hline 50 & Dec. 22,1965 & 19 & 41 & 23 & $58.4 \mathrm{~N}$ & $153.0 \mathrm{~W}$ & $50 \mathrm{R}$ & $6 \frac{3}{4}-7$ & PAS & 33.5 & $\mathrm{R}_{1}$ & 0.07 \\
\hline 51 & $\operatorname{Jan} .15,1966$ & 11 & 59 & 59 & $59.5 \mathrm{~N}$ & $144.6 \mathrm{~W}$ & 33 & 5.0 & PAS & 30.7 & $\mathrm{R}_{1}$ & -0.90 \\
\hline 52 & Jan. 20 & 04 & 27 & 45 & $15.1 \mathrm{~S}$ & $168.0 \mathrm{E}$ & 28 & 5.5 & PAS & 88.5 & $\mathbf{R}_{1}$ & -0.48 \\
\hline 53 & Jan. 22 & 14 & 27 & 08 & $56.0 \mathrm{~N}$ & $153.7 \mathrm{~W}$ & $33 \mathrm{R}$ & 6 & PAS & 32.7 & $\mathrm{R}_{1}$ & -0.23 \\
\hline 54 & Feb. 4 & 10 & 39 & 12 & $15.9 \mathrm{~S}$ & $167.9 \mathrm{E}$ & 190 & 5.6 & PAS & 86.1 & $\mathbf{R}_{1}$ & 0.28 \\
\hline & & & & & & & & & & & $\mathbf{R}_{\mathbf{2}}$ & 0.38 \\
\hline 55 & Feb. 5 & 02 & 01 & 48 & $39.2 \mathrm{~N}$ & $22.0 \mathrm{E}$ & 38 & $6 \frac{1}{4}$ & PAS & 98.2 & $\mathbf{R}_{\mathrm{I}}$ & 0.42 \\
\hline 56 & Feb. 5 & 15 & 12 & 29 & $26.1 \mathrm{~N}$ & $103.1 \mathrm{E}$ & 15 & 5.7 & PAS & 108.4 & $\mathbf{R}_{1}$ & 0.36 \\
\hline 57 & Feb. 9 & 04 & 40 & 28 & $56.7 \mathrm{~S}$ & $25.7 \mathrm{~W}$ & 27 & $5 \frac{1}{2}$ & PAS & 110.1 & $\mathrm{R}_{\mathrm{i}}$ & 0.52 \\
\hline & & & & & & & & & & & $\mathbf{R}_{2}$ & 0.77 \\
\hline & & & & & & & & & & & $\mathrm{R}_{3}$ & 0.45 \\
\hline 58 & Feb. 10 & 14 & 21 & 11 & $20.8 \mathrm{~N}$ & $146.3 \mathrm{E}$ & 43 & $6 \frac{1}{2}$ & PAS & 83.0 & $\mathrm{R}_{1}$ & 0.39 \\
\hline 59 & Feb. 16 & 08 & 18 & 27 & $17.7 \mathrm{~S}$ & $167.9 \mathrm{E}$ & 31 & $6 \frac{1}{2}$ & PAS & 87.1 & $\mathbf{R}_{\mathrm{L}}$ & 0.31 \\
\hline & & & & & & & & & & & $\mathbf{R}_{2}$ & 0.37 \\
\hline 60 & Feb. 22,1966 & 05 & 02 & 37 & $5.4 \mathrm{~S}$ & $151.5 \mathrm{E}$ & $28 \mathrm{R}$ & $6 \frac{3}{4}$ & PAS & 93.3 & $\mathbf{R}_{1}$ & 0.37 \\
\hline 61 & March 6 & 18 & 01 & 50 & $24.1 \mathrm{~S}$ & $176.9 \mathrm{~W}$ & $33 \mathbf{R}$ & 5,1 & PAS & 80.4 & $\mathbf{R}_{1}$ & -0.61 \\
\hline 62 & March 11 & 07 & 54 & 17 & $55.2 \mathrm{~s}$ & $126.6 \mathrm{~W}$ & $33 \mathrm{R}$ & 5.0 & PAS & 89.3 & $\mathrm{R}_{1}$ & -1.08 \\
\hline 63 & March 12 & 16 & 31 & 22 & $24.1 \mathrm{~N}$ & $122.6 \mathrm{E}$ & 63 & $7 \frac{1}{2}-7 \frac{3}{4}$ & PAS & 98.2 & $\mathrm{R}_{2}$ & 1.80 \\
\hline & & & & & & & & & & & $\mathrm{R}_{3}$ & 1.91 \\
\hline 64 & March 13 & 17 & 58 & 36 & $55.0 \mathrm{~S}$ & $126.4 \mathrm{~W}$ & $33 R$ & 5.2 & PAS & 89.1 & $R_{1}$ & -0.66 \\
\hline 65 & Mareh 20 & 01 & 42 & 50 & $0.6 \mathrm{~N}$ & $30.2 \mathrm{E}$ & $36 \mathrm{R}$ & $6 \frac{a}{4}-7$ & PAS & 134.5 & $\mathrm{R}_{1}$ & 1.42 \\
\hline 66 & March 22 & 08 & 19 & 34 & $37.5 \mathrm{~N}$ & $115.1 \mathbf{E}$ & 33 & $66_{4}^{3}-7$ & PAS & 93.2 & $\mathrm{R}_{\mathrm{t}}$ & $0.8 \mathrm{~B}$ \\
\hline & & & & & & & & & & & $\mathrm{R}_{2}$ & 0.60 \\
\hline 67 & April 6 & 02 & 59 & 02 & $45.8 \mathrm{~S}$ & $96.1 \mathrm{E}$ & 33 & 6 & PAS & 151.5 & $\mathrm{R}_{1}$ & 0.46 \\
\hline & & & & & & & & & & & $\mathrm{R}_{2}$ & 0.20 \\
\hline 68 & April 10 & 16 & 36 & 15 & $31.5 \mathrm{~S}$ & $71.2 \mathrm{~W}$ & $64 \mathrm{R}$ & 6 & PAS & 78.9 & $\mathbf{R}_{1}$ & -0.34 \\
\hline 69 & April 16 & 01 & 27 & 15 & $5 \% .0 \mathrm{~N}$ & $153.6 \mathrm{~W}$ & $33 \mathrm{R}$ & $6 \frac{1}{4}$ & PAS & 33.1 & $\mathbf{R}_{1}$ & 0.03 \\
\hline 70 & A pril 20 & 06 & 00 & 39 & $18.9 \mathrm{~N}$ & $146.8 \mathrm{E}$ & $33 \mathrm{R}$ & 5.0 & PAS & 83.6 & $R_{1}$ & -0.31 \\
\hline 71 & April 22 & 23 & 27 & 21 & $57.5 \mathrm{~N}$ & $152.1 \mathrm{~W}$ & 22 & 5.3 & PAS & 32.7 & $\mathbf{R}_{1}$ & -0.41 \\
\hline 72 & April 23 & 00 & 09 & 34 & $0.9 \mathrm{~S}$ & $122.4 \mathrm{E}$ & 45 & $6 \frac{3}{4}$ & PAS & 113.5 & $\mathbf{R}_{1}$ & 1.27 \\
\hline & & & & & & & & & & & $\mathrm{R}_{2}$ & 1.16 \\
\hline & & & & & & & & & & & $\mathrm{R}_{\mathrm{a}}$ & 1.28 \\
\hline 73 & April 23 & 08 & 56 & 46 & $0.5 \mathrm{~s}$ & $122.2 \mathrm{E}$ & 79 & 6 & PAS & 113.9 & $\mathbf{R}_{2}$ & 0.10 \\
\hline & & & & & & & & & & & $\mathrm{R}_{2}$ & -0.04 \\
\hline
\end{tabular}


TABLE 2-Continued

\begin{tabular}{|c|c|c|c|c|c|c|c|c|c|c|c|c|}
\hline \multirow{2}{*}{ 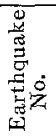 } & \multirow{2}{*}{ Date } & \multicolumn{3}{|c|}{ Origin Time } & \multirow{2}{*}{ Lat. } & \multirow{2}{*}{ Long. } & \multirow{2}{*}{$\underset{(\mathrm{km})}{\text { Depth }}$} & \multirow{2}{*}{$\begin{array}{l}\text { Magn- } \\
\text { tude }\end{array}$} & \multirow{2}{*}{ Station } & \multirow{2}{*}{$\begin{array}{c}\text { Epi- } \\
\text { central } \\
\text { Distance } \\
\text { (degrees) }\end{array}$} & \multirow{2}{*}{$\mid \begin{array}{c}\text { Signal } \\
\text { Used }\end{array}$} & \multirow{2}{*}{$\underset{A_{90^{*}}}{\log }$} \\
\hline & & h & $\mathrm{m}$ & s & & & & & & & & \\
\hline 74 & May 1,1966 & 16 & 22 & 56 & $8.5 \mathrm{~S}$ & $74.3 \mathrm{~W}$ & $165 \mathrm{R}$ & $6 \frac{3}{4}$ & PAS & 59.4 & $\begin{array}{l}\mathrm{R}_{1} \\
\mathrm{R}_{2}\end{array}$ & $\begin{array}{l}0.60 \\
0.65\end{array}$ \\
\hline 75 & May 15 & 14 & 46 & 07 & $51.5 \mathrm{~N}$ & $178.4 \mathrm{~W}$ & $31 \mathrm{R}$ & $5 \frac{3}{1}-6$ & PAS & 46.1 & $\mathrm{R}_{1}$ & 0.05 \\
\hline 76 & May 19 & 07 & 06 & 27 & $54.1 \mathrm{~N}$ & $164.1 \mathrm{~W}$ & 28 & 6 & PAS & 37.7 & $R_{1}$ & 0.25 \\
\hline 77 & May 20 & 09 & 14 & 49 & $13.9 \mathrm{~N}$ & $146.1 \mathrm{E}$ & 66 & $6 \frac{1}{4}-6 \frac{1}{2}$ & PAS & 87.0 & $\mathbf{R}_{1}$ & -0.12 \\
\hline 78 & June 1 & 11 & 47 & 33 & $23.4 \mathrm{~S}$ & $174.9 \mathrm{~W}$ & 24 & 5.0 & PAS & 78.6 & $\mathrm{R}_{1}$ & -0.37 \\
\hline 79 & June 2 & 03 & 27 & 53 & $51.1 \mathrm{~N}$ & $176.0 \mathrm{E}$ & $41 R$ & 6 & PAS & 49.6 & $\mathrm{R}_{1}$ & -0.43 \\
\hline 80 & June 4 & 23 & 48 & 18 & $46.5 \mathrm{~N}$ & $152.5 \mathrm{E}$ & $27 \mathrm{R}$ & $5_{4}^{3}-6$ & PAS & 65.8 & $\mathrm{R}_{1}$ & 0.00 \\
\hline 81 & June 6 & 07 & 46 & 16 & $36.3 \mathrm{~N}$ & $71.2 \mathrm{E}$ & $225 \mathrm{R}$ & $6 \frac{3}{4}$ & PAS & 109.4 & $\begin{array}{l}\mathrm{R}_{1} \\
\mathrm{R}_{2} \\
\mathrm{R}_{3}\end{array}$ & $\begin{array}{l}1.25 \\
1.34 \\
1.36\end{array}$ \\
\hline 82 & June 15 & 00 & 59 & 46 & $10.4 \mathrm{~S}$ & $160.8 \mathrm{E}$ & 31 & $7 \frac{1}{2}$ & PAS & 88.5 & $\begin{array}{l}\mathrm{R}_{1} \\
\mathrm{R}_{2} \\
\mathrm{R}_{3}\end{array}$ & $\begin{array}{l}1.81 \\
2.13 \\
1.57\end{array}$ \\
\hline 83 & June 15 & 01 & 32 & 56 & $10.2 \mathrm{~S}$ & $161.1 \mathrm{E}$ & $33 R$ & $7 \frac{1}{4}$ & PAS & 88.1 & $\begin{array}{l}\mathbf{R}_{1} \\
\mathbf{R}_{2} \\
\mathbf{R}_{3}\end{array}$ & $\begin{array}{l}1.58 \\
1.64 \\
1.54\end{array}$ \\
\hline 84 & July 4, 1966 & 18 & 33 & 36 & $51.7 \mathrm{~N}$ & $179.9 \mathrm{E}$ & 13 & $6 \frac{3}{4}-7$ & PAS & 47.1 & $\begin{array}{l}R_{1} \\
R_{2} \\
R_{3}\end{array}$ & $\begin{array}{l}1.49 \\
1.66 \\
1.60\end{array}$ \\
\hline 85 & July 19 & 01 & 40 & 54 & $56.2 \mathrm{~N}$ & $164.9 \mathrm{E}$ & 18 & $6-6 \frac{1}{4}$ & PAS & 55.4 & $\mathbf{R}_{1}$ & 0.73 \\
\hline 86 & Aug. 1 & 21 & 03 & 00 & $30.0 \mathrm{~N}$ & $68.7 \mathrm{E}$ & $33 \mathbf{R}$ & $6 \frac{3}{4}$ & PAS & 115.9 & $\begin{array}{l}\mathrm{R}_{1} \\
\mathrm{R}_{2}\end{array}$ & $\begin{array}{l}1.65 \\
1.20\end{array}$ \\
\hline 87 & Aug. 7 & 02 & 13 & 05 & $50.6 \mathrm{~N}$ & $171.3 \mathrm{~W}$ & $39 R$ & $6 \frac{3}{4}$ & PAS & 41.6 & $\mathbf{R}_{1}$ & 0.74 \\
\hline 88 & Aug. 19 & 12 & 22 & 10 & $39.2 \mathrm{~N}$ & $41.7 \mathrm{E}$ & $26 \mathrm{R}$ & 7 & PAS & 104.7 & $\mathbf{R}_{1}$ & 1.84 \\
\hline
\end{tabular}

mined in some cases at Pasadena and in other cases both at Pasadena and Berkeley. For earlier earthquakes recorded on Milne-Shaw instruments, the magnitudes are taken from Richter (1958).

The basic data obtained are mantle Rayleigh wave ground amplitudes (vertical component) at a period of 100 seconds corrected to an epicentral distance of $90^{\circ}$. Amplitudes were read direetly from the records and corrected for instrument response and distance effects. See Figure 1 for an example of a measurement of amplitude. Observations on horizontal component instruments were corrected for azimuth and multiplied by $\frac{4}{3}$ to convert to equivalent vertical amplitudes. For magnitudes less than 6 the data are biased toward higher amplitudes since the amplitudes for some smaller events may be too small to be recorded at large distances. However, all earthquakes with $M \geqq 6$ which occurred during the period represented by the data for the CIT ULP instrument with a gain of 1600 , generated mantle waves which were recorded and included in the data. Thus there is no reason to expect bias for $M \geqq 6$.

The distance correction consists of two factors. The first factor consists of the following: (1) A correction for dispersion. This is based on theoretical seismograms corresponding to the known group velocity curve for mantle waves. This correction is proportional to the square root of distance for distances larger than about $160^{\circ}$. (2) A correction for geometric spreading on a sphere. This is proportional to the square root of the sine of the distance in degrees, except near the epicenter and antipodes. Near the epicenter and antipodes it is proportional to $\sqrt{\pi n / 2} / P_{n}(\cos \Delta)$, where $P_{n}(\cos \Delta)$ is the Legendre polynomial of order $n$, and $n+\frac{1}{2}$ is equal to the 
radius of the earth divided by the wavelength. However, measurement of amplitude within a wavelength of the epicenter or antipodes will probably not be reliable since waves arriving from many directions interfere. (3) A correction for attenuation. This is given by $\exp (\pi x / Q U T)$ where $Q$ is taken as 145 (Ben-Menahem, 1965), $x$ is the distance, $U$ the group velocity, and $T$ the period (100 seconds).

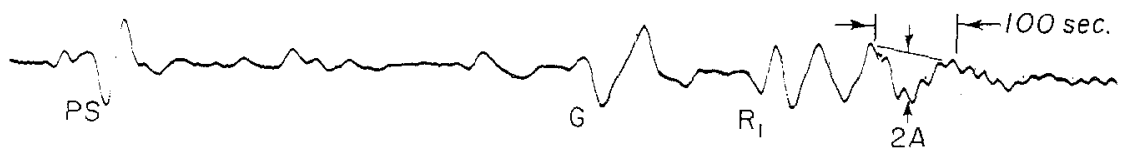

FIG. 1. Seismogram illustrating mantle Rayleigh wave train $R_{1}$ and method of measuring amplitude. 1 his seismogram is for the ? onga Island earthquake of 21 December $1959(M=6)$ recorded on a CIT UL.P instrument at Pasadena (Gilman, 1960).

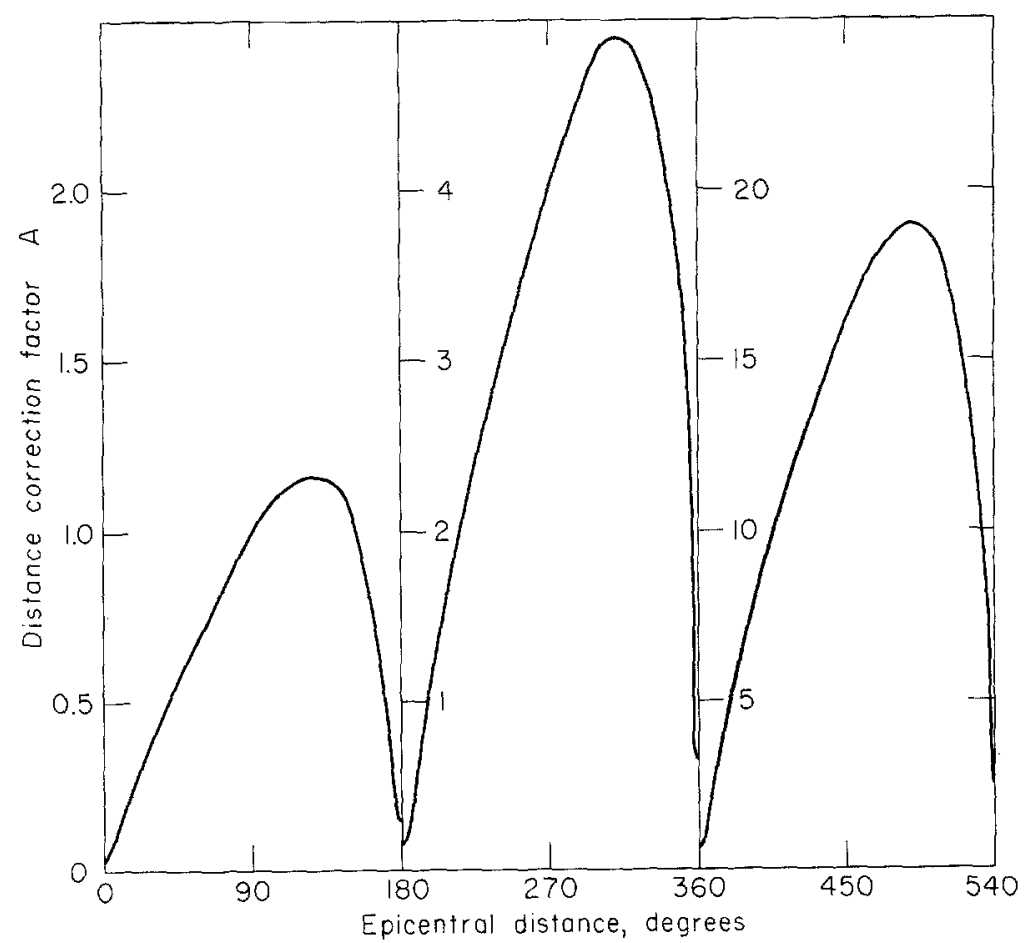

FIG. 2a. Distance correction factor, A, for 100 second period mantle Rayleigh waves.

The product of these corrections, $A$, is given as a function of distance in Figure 2a.

A further empirical correction factor was determined by numerous correlations of $R_{1}$ to $R_{3}$ and $R_{1}$ to $R_{2}$. This empirical factor corrects for effects such as:

(1) Change in the shape of the spectrum as a function of distance. This will change the ratio of the observed trace amplitude to the true spectral amplitude.

(2) Uncertainty in the appropriate $Q$ value and deviations from an exact exponential attenuation.

(3) Abnormal dispersion, due in part to scattering and refraction. 
(4) The effect of the shape of the response curve for a particular type of instrument. This effects the distance dependence of trace amplitude at distances of the order of $100^{\circ}$ and less. The empirical correction factor obtained here in a strict sense only applies to the CIT ULP instrument; however, the effect is small and probably nearly the same for the other instruments used in this study.

This empirical correction factor, $B$, is determined to be a factor of 1.6 at $\Delta=270^{\circ}$ and 2.3 at $\Delta=450^{\circ}$. It is approximated as a linear function of distance and shown in Figure 2b.

The logarithms of the ground amplitudes in microns, corrected to a distance of

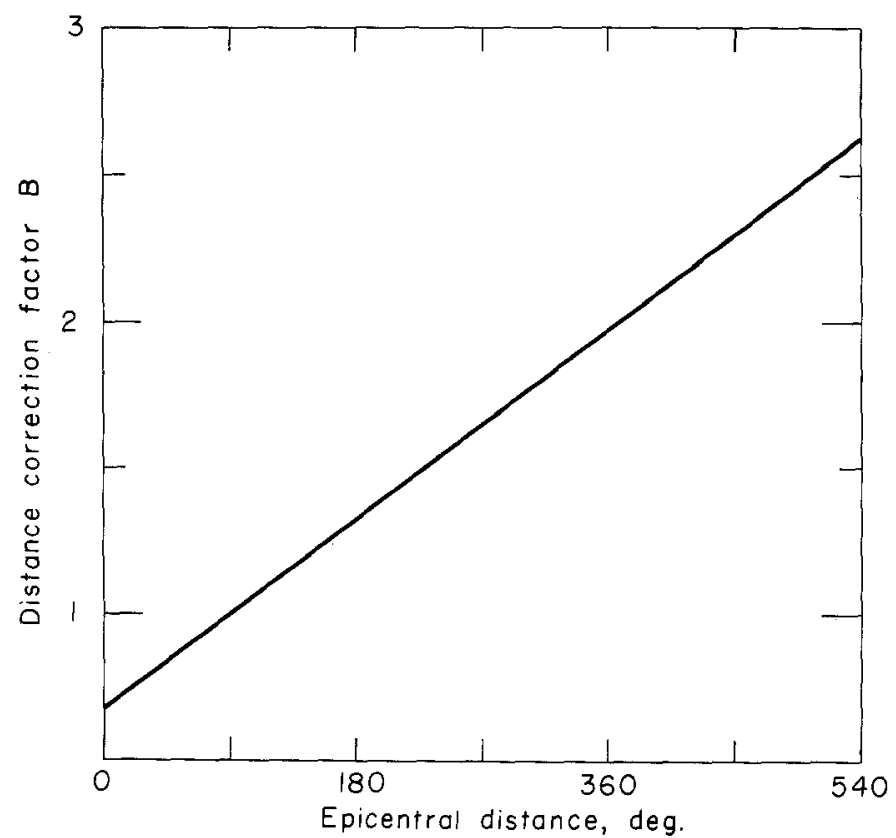

FIG. 2b. Distance correction factor, $B$, for 100 second period mantle Rayleigh waves.

$90^{\circ}$, are shown in Table 2 and plotted as a function of magnitude in Figure 3. Most of the earthquakes used have depths less than $60 \mathrm{~km}$, but the few deeper ones, with depths up to $225 \mathrm{~km}$, give results comparable to the shallow shocks. For these deeper quakes the magnitude $M$ was determined from body waves.

\section{Theoretical Curve}

Large shallow earthquakes are often associated with long surface breaks and long aftershock zones. Tocher (1958), Iida (1959; 1965) and Albee and Smith (1966), have given curves of approximate length of faulting or length of aftershock zone versus magnitude. The propagation of the seismic source should control the ratio of the amplitudes of 100 second waves to 20 second waves and thus the excitation of mantle waves as a function of magnitude. The effect of a propagating source can be derived quite simply (Benioff, 1955; Ben-Menahem, 1961; Haskell, 1963). The effect is to multiply the amplitude spectrum by the directivity function, $\sin X / X$, (BenMenahem, 1961) where: 


$$
X=\frac{-\pi b}{c T}\left(\frac{c}{v}-\cos \phi\right)
$$

In this expression $b$ is the fault length, $c$ the phase velocity, $T$ the period, $v$ the fault propagation velocity, and $\phi$ the angle between the direction of fault propagation and the direction to the recording station.

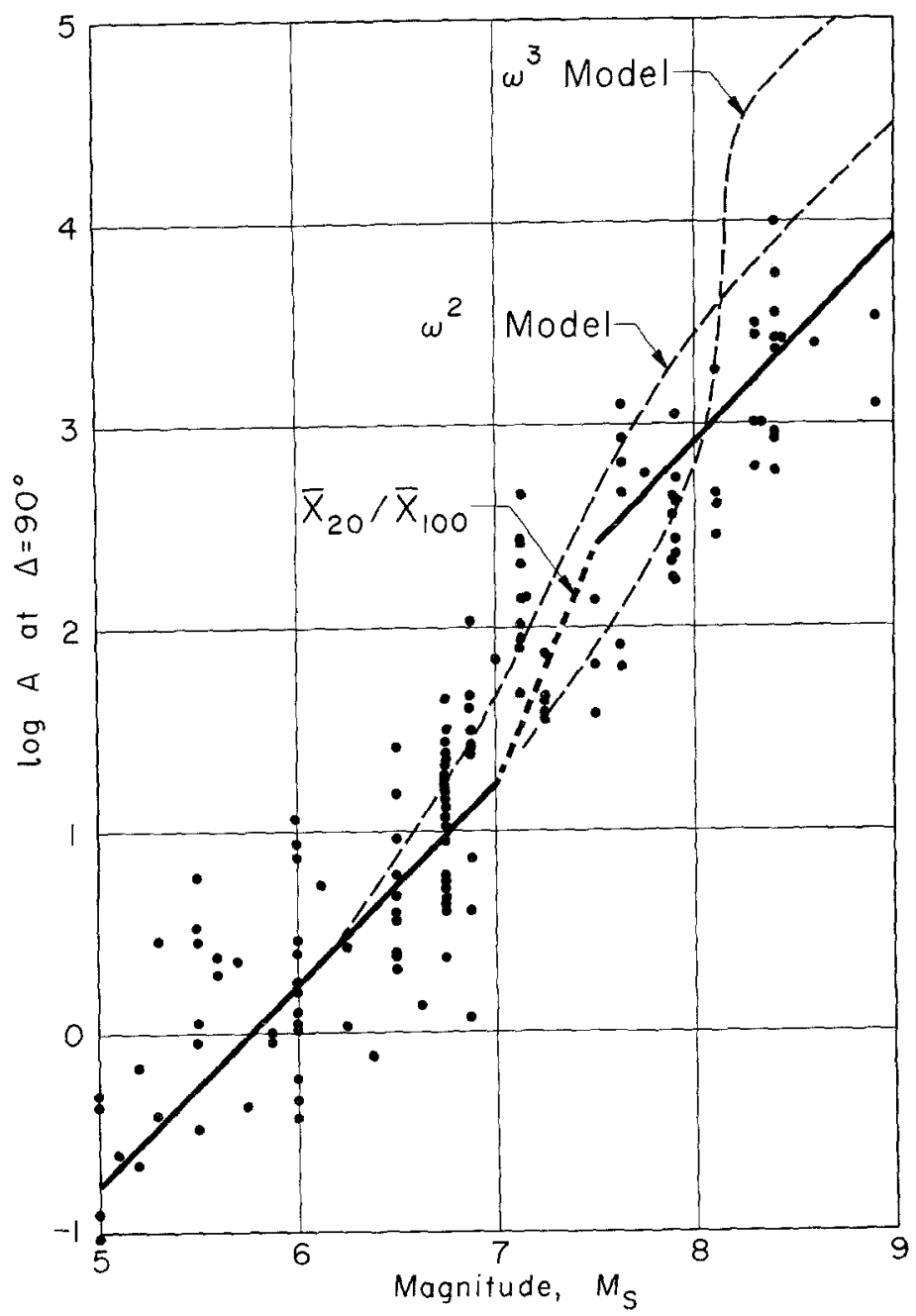

FIG. 3. Logarithm to the base 10 of the amplitude of vertical ground motion (in microns) of 100 second period mantle Rayleigh waves at a distance of $90^{\circ}$, plotted as a function of magnitude $M_{s}$. The curve labeled $X_{20} / X_{100}$ is based on a traveling source model and the curves labeled $\omega^{2}$ and $\omega^{3}$ are based on models discussed by Aki (1967).

In order to obtain an approximate theoretical curve for the amplitudes of 100 second mantle waves as a function of magnitude, we proceed as follows:

We assume that the earthquake magnitude is directly proportional to the amplitude of 20 second surface waves as implied by the surface wave magnitude scale. We then compute the ratio of the directivity function for 100 second mantle waves 
to that for 20 second surface waves as a function of fault length (or length of aftershock zone), assuming further that $v=3 \mathrm{~km} / \mathrm{sec}$,

$$
R(b)=\frac{\sin X_{100} / X_{100}}{\sin X_{20} / \bar{X}_{20}}
$$

This function in general will have zeros and infinities in it due to the sine functions, but by making a large number of observations we will tend to average out these effects. We shall assume that the erratic behavior of $R(b)$ is smoothed by averaging the scatter in the observed data, so that $\overline{R(b)}_{2} \bar{X}_{20} / \bar{X}_{100}$ (= 5 for large fault lengths since the average value of $\cos \phi$ is zero).

Having $\overline{R(b)}$ as a function of fault length and having a relationship between fauit length and magnitude, we obtain a theoretical curve for excitation of mantle waves as a function of magnitude. The curve given in Figure 3 (labelled $\bar{X}_{20} / \bar{X}_{100}$ ) is determined from relationships between fault length and magnitude given by Tocher (1958) and Iida $(1958 ; 1965)$ by smoothing into three straight line segments. The portion of the curve above magnitude 7 reflects the interference effects of the propagating rupture. The absolute value of the curve agrees at $M=6$ with the theoretical amplitude ratio of 100 second to 20 second Rayleigh waves for a point couple in a layer over a half-space (computed from the theory of Yanovskaya, 1958, assuming a $Q$ of 400 for 20 second Rayleigh waves).

Aki (1967) has recently used a statistical or incoherent source model proposed by Haskell (1966) to derive a scaling law for seismic spectrum. This model is referred to as the $\omega^{3}$ model since it predicts the amplitude spectrum for an earthquake will decrease as $1 / \omega^{3}$ at high frequencies. Aki proposed another model based on similar considerations but having a decrease in amplitude proportional to $1 / \omega^{2}$ at high frequencies. This model is referred to as the $\omega^{2}$ model and agrees with the data presented by Aki much better than does the $\omega^{3}$ model. From curves presented by Aki we can directly obtain the 100 second to 20 second amplitude ratio as a function of magnitude for the $\omega^{2}$ and $\omega^{3}$ models. The corresponding curves are shown in Figure 3.

\section{Discussion}

The data shown in Figure 3 indicate a range of amplitude of somewhat greater than a factor of ten at any given magnitude. This is considerably less than the range of amplitudes of 20 second surface waves for a given local earthquake magnitude based on waves of about $1 \mathrm{sec}$ period (Brune et al, 1963), suggesting that mantle waves are a more reliable measure of earthquake size than shorter period waves. This is not unexpected since these very long wavelengths should not be as strongly influenced by interference and local effects. A mantle wave magnitude scale based on the solid curve in Figure 3 would thus have some merit.

The data agree well with the $X_{20} / X_{100}$ curve, indicating that on the average earthquakes can be adequately modelled by a propagating rupture, with the length of rupture approximately as given by Tocher's and Iida's curves.

The data agree better with the $\omega^{2}$ than with the $\omega^{3}$ model, but nevertheless lie significantly below both models for $M>8.0$. For the $\omega^{3}$ model the scale effect increases sharply at $M=8.0$ and for $M=8.4$ gives amplitudes of 100 sec waves 
25 times greater than the average of the observed data. For the $\omega^{2}$ model the scale effect increases the curve gradually from $M=6$ and it gives amplitudes about 4 times higher than the mean of the data beyond $M=8.0$. Thus the increase in the $100 \mathrm{sec}$ to $20 \mathrm{sec}$ amplitude ratio with increasing magnitude is much less than predicted by the $\omega^{3}$ model and somewhat less than predicted by the $\omega^{2}$ model. This may be due to the lack of strict similarity between large earthquakes and smaller ones. Tocher (1958) interpreted his data as indicating that for large earthquakes the fault length increases at a much faster rate than fault depth, thus violating the rule of similarity assumed by Aki. The data might also be explained by the type of incoherency in the source region. The data in Figure 3 indicate that the scale effect on the amplitude ratio of 100 to 20 second waves from magnitude 6 to magnitude 8.4 is about a factor of 5 in agreement with the $\bar{X}_{20} / \bar{X}_{100}$ model. A factor of 20 is predicted by the $\omega^{2}$ model and a factor of 125 by the $\omega^{3}$ model. This suggests that if a scaling law appropriate for this frequency and magnitude range is to be constructed it would be of the $\omega$ type, i.e., the spectral amplitudes for a given earthquake would be proportional to $1 / \omega$. If we accept Aki's $\omega^{2}$ model for shorter periods then the data indicate that a different type of incoherency occurs for periods of about 20 seconds. Haskell (personal communication) has pointed out that the displacement spectrum at high frequencies must fall off faster than $\omega^{-3 / 2}$ in order to keep the energy integral finite.

\section{ACKNOWLEDGMENTS}

One of the authors (Chi-Yu King) was a post-doctoral fellow at the Califormia Institute of Technology while participating in the study. Special thanks are due to Mr. Robert Eppley of the U. S. Coast and Geodetic Survey who made many of the amplitude readings from Columbia ULP records and to Mr. Ralph Gilman who provided records from the Pasadena (PAS) ULP instruments and assisted in the preparation of the paper. The authors are indebted to Professor Keiiti Aki for critically reading the manuscript and offering constructive criticism. Professor Stewart Smith provided records and calibration curves for the Isabella (ISA) station. This research was supported in part by the U. S. Coast and Geodetic Survey under contract CGS 1091 (G) while the first author was at Lamont Geological Observatory, the National Science Foundation under grant GP 2806, and the Air Force Office of Scientific Research, Office of Aerospace Research, United States Air Foree, under AFOSR contract AF-49(638)-1337.

\section{REFERENCES}

Aki, K. (1967). Scaling law of seismic spectrum, J. Geophys. Res. 72, 1217-1231.

Albee, A. L. and J. L. Smith (1966). Earthquake characteristics and fault activity in southern California, engineering geology of southern California, Assoc. Engineering Geologist, 9-33.

Benioff, H. (1955). Mechanism and strain characteristics of the White Wolf fault as indicated by the aftershock sequence, Calif. Div. Mines, Bull. 171, 199-202.

Benioff, H. (1959). Fused-quartz extensometer for secular, tidal and seismic strains, Bull. Geol. Soc. Am. 70, 1019-1032.

Ben-Menahem, A. (1961). Radiation of seismic surface-waves from finite moving sources, Bull. Seism. Src. Am. 51, 401-435.

Ben-Menahem, A. (1965). Observed attenuation and Q values of seismic surface waves in the upper mantle, J. Geophys, Res. 70, 4641-4651.

Brune, J. N. (1963). Use of surface wave rejection filters to record mantle waves of lower order, Earthquake Notes, 34, 73.

Brune, J. N., A. Espinosa and J. E. Oliver (1963). Relative excitation of surface waves by earthquakes and underground explosions in the California-Nevada region, J. Geophys. Res. 68, 3501-3513. 
Gilman, R. (1960). Report on some experimental long-period seismographs, Bull. Seism. Soc. Am. 50, 553-559.

Gutenberg, B. (1945). Amplitudes of surface waves and magnitudes of shallow earthquakes, Bull. Seism. Soc. Am. 35, 3-12.

Gutenberg, B., and C. F. Richter (1956). Magnitude and energy of earthquakes, Ann. Geofis. $9,1-15$.

Haskell, N. A. (1963). Radiation pattern of Rayleigh waves from a fault of arbitrary dip and direction of motion in a homogeneous medium, Bull. Seism. Soc. Am. 53, 619-642.

Haskell, N. A. (1964). Total energy and energy spectral density of elastic wave radiation from propagating faults, Bull. Seism. Soc. Am. 54, 1811-1841.

Haskell, N. A. (1966). Total energy and energy spectral density of elastic wave radiation from propagating faults, Part II. A statistical source modej, Bull. Seism. Soc. Am. 56, 125-140.

Iida, K. (1959). Earthquake energy and earthquake fault, J. Earth Sci., Nagoya Univ. 7, 98-107.

Iida, K. (1965). Earthquake magnitude, earthquake fault, and source dimension, $J$. Earth. Sci., Nagoya Univ. 13, 115-132.

Richter, C. F. (1958). Elementary Seismology, W. H. Freeman and Co., San Francisco.

Sutton, G. and J. Oliver (1959). Seismographs of high amplification at long periods, Annales Geophys. 15, 423.

Tocher, D. (1958). Earthquake energy and ground breakage, Bull. Seism. Soc. Am. 48, 147-153.

Wyss, M. and J. N. Brune (1967). The Alaska earthquake of 28 March 1964: A complex multiple rupture, Bull. Seism. Soc. Am. 57, 1017-1023.

Yanovskaya, T. B. (1958). On the determination of the dynamic parameters of the focus hypocenter of an earthquake from records of surface waves, Bull. (Izvestiya), Acad. of Sciences, U.S.S.R., Geophys. Series, No. 3, 289-301.

Seismological Laboratory

California Institute of Technology

Pasadena, California

Division of Geological Sciences Contribution No. 1462.

Lamont Geological Observatory

Columbia University

Palisades, New York

Contribution No. 1126

Manuscript received May 16, 1967. 